\title{
Applications Of Tunable Mid-Infrared Plasmonic Square-Nanoring Resonator Based On Graphene Nanoribbon
}

Morteza Janfaza

University of Sistan and Baluchestan

Mohammad Ali Mansouri-Birjandi ( $\sim$ mansouri@ece.usb.ac.ir)

University of Sistan and Baluchestan https://orcid.org/0000-0002-8378-364X

Alireza Tavousi

Velayat University

\section{Research Article}

Keywords: Demultiplexer, Graphene nanoribbons, Surface plasmons, Square-nanoring, Power splitter

Posted Date: June 7th, 2021

DOl: https://doi.org/10.21203/rs.3.rs-497948/v1

License: (1) This work is licensed under a Creative Commons Attribution 4.0 International License.

Read Full License

Version of Record: A version of this preprint was published at Plasmonics on September 28th, 2021. See the published version at https://doi.org/10.1007/s11468-021-01538-y. 


\title{
Applications of Tunable Mid-Infrared Plasmonic Square- Nanoring Resonator Based on Graphene Nanoribbon
}

\author{
Morteza Janfaza ${ }^{1}$, Mohammad Ali Mansouri-Birjandi ${ }^{1, *}$ and Alireza Tavousi ${ }^{2}$ \\ ${ }^{1}$ Faculty of Electrical and Computer Engineering, University of Sistan and Baluchestan (USB), P. O. \\ Box 9816745563 , Zahedan, Iran \\ ${ }^{2}$ Department of Electrical Engineering, Velayat University, P.O. Box 9911131311, Iranshahr, Iran \\ *Corresponding author: mansouri@ece.usb.ac.ir
}

\begin{abstract}
In this work, different structures are designed based on graphene square-nanoring resonator (GSNR) and simulated by the three-dimensional finite-difference time-domain (3DFDTD) method. Depending on the location and number of graphene nanoribbons (GNR), the proposed structures can be utilized as a band-pass filter, wavelength demultiplexer, or power splitter in the mid-infrared (MIR) wavelengths. The tunability of the suggested assemblies is easily controlled by changing the dimensions and/or the chemical potential of the GSNRs. Benefiting from the nanoscale and ultra-compact GNRs, these structures can be proposed as basic blocks for optical computing and signal processing in the MIR region.
\end{abstract}

Keywords: Demultiplexer, Graphene nanoribbons, Surface plasmons, Square-nanoring, Power splitter. 


\section{Introduction}

Surface plasmon polaritons (SPPs), the electromagnetic waves propagating at the conductordielectric interfaces, are known as reliable and promising phenomena to overcome diffraction limit and realize devices in sub-wavelength and nanometer scales [1-3]. Diverse plasmonic devices have been designed and proposed based on controlling and manipulation of the propagation performance of SPPs at the metal-dielectric surfaces. However, major barriers, such as high loss and the lack of dynamic tunability, limit these plasmonic structures to be employed and implemented in practical applications [4-7]. Graphene, due to its unique electrical and optical properties, has become an attractive material for researchers and can be used as a promising platform for photonics and plasmonics, in recent years. This is due to the ability of the graphene surface, which can support SPPs, known as graphene surface plasmon polaritons (GSPPs) [8-10], leading to significant progress observed in the field of graphene plasmonics.

Graphene is a two-dimensional material that behaves like a single atom layer of metal since it has a negative relative permittivity in the mid-infrared (MIR) wavelength range [11, 12]. The surface conductivity of graphene has a wide range of tunable characteristics, yields only via the Fermi level adjustment of the material through electrostatic gating or chemical doping, which makes it widely used in tunable and high-performance optical devices [13]. Including graphene in the design and fabrication of integrated nano-plasmonic and nanophotonic devices brings many advantages compared to noble metals. To name a few [1416]: (1) Ultra-thin thickness of graphene (much thinner than any metallic layer) makes it suitable to be used as waveguides in the supported wavelength ranges. (2) It has low ohmic losses at high-doped levels. (3) The large effective index of graphene structures in the MIR range, leading to high compression of the light and ultra-short local wavelengths within the material is a key to overcome the diffraction limit of the light compared to the traditional optical devices. In other words, SPP waves in graphene have wave vectors nearly 100 times larger than their free space counterparts, which makes the design of graphene-based optical devices easier, because the dimensions of the components are below the diffraction limit. (4) The conductivity of the material can be changed (tuning the chemical potential) by either doping or gate voltage (electrostatic gating). This makes the device to be set and calibrated without the need to re-fabrication the structure. (5) GSSPs have longer propagation lengths in the MIR region than metallic surface plasmon waves. 
Over the two past decades, there has been extensive research into a variety of graphenebased plasmonic devices such as modulators [17, 18], switches [19, 20], filters [21-23], power splitter [24, 25], metamaterials [26, 27], metasurfaces [28, 29], as well as graphenebased plasmonic induced transparency (PIT) and reflection-induced (PIR) effects [30-32]. Most of these devices work based on graphene nanoribbons (GNRs), formed by reducing the width of the graphene flake. When the width of the graphene ribbon is reduced to less than a few tens of nanometers, the waveguide mode disappears and only edge mode at the rims of the graphene strips can be excited vertically and laterally. In addition, the edge modes supported by GNRs have two distinct odd and even edge mode participation $[33,34]$. When the width of a GNR is a few tens of nanometers, the odd edge modes vanish and we only have the even modes. This even edge mode has great transmission property, especially in the field of filter design. Another advantage of GNR in such devices is the relatively higher effective refractive index of the edge modes compared to waveguide modes, and consequently, a much smaller effective wavelength [35,36]. This makes the GNR-based plasmonic device ultra-compact with a small footprint.

In this paper, using these great advantages of GNR in the MIR wavelengths, a structure is proposed to be simultaneously used as a filter, demultiplexer, and power splitter, which could reduce the device area for some particular applications, where two of the devices may be needed simultaneously in associated working frequency. To this end, three different structures are presented based on GSNR resonators, numerically analyzed using the 3DFDTD method. Depending on the number and location of output GNR waveguides (output port), these structures may have practical applications such as filter, wavelength demultiplexer, and power splitter, which all them are fundamental elements in nanophotonic and nanoplasmonic integrated circuits. In all of these structures, GNRs with a width of a few nanometers are used, which makes the structures presented simple in terms of design and fabrication, and most importantly, ultra-compact to be utilized in nanoscale devices at MIR functional region. The organization of the remains of the paper is as follows: In Section 2, the proposed structures and their various parameters are explained along with the theories and methods. Section 3 presents the results and discussions related to the proposed structures and finally, the conclusion is made in Section 4. 


\section{The Structural Properties and Theoretical Analysis Method}

The schematic diagram of the proposed structures with the detailed geometrical parameters is shown in Fig. 1. All the three structures shown in figure (a-c) use identical graphene nanoribbons (GNRs) as incoming and outgoing waveguides, while a graphene-based square nanoring (GSNR) resonator completes the role of the filter (in Fig. 1(a)), demultiplexer (Fig. 1(b)), or the power splitter (Fig. 1(c)), depending on the location and the layout of the output GNR(s). The geometrical parameters include $\mathrm{L}_{1}=100 \mathrm{~nm}$ and $\mathrm{W}_{\mathrm{s}}=20 \mathrm{~nm}$ (the side lengths and the width of the GSNR), $\mathrm{L}_{2}=100 \mathrm{~nm}$ and $\mathrm{L}_{3}=150 \mathrm{~nm}$ (the distances between the considered ports, that is where the detectors are placed, and the edge of the GNRs), $\mathrm{W}_{\mathrm{n}}=20 \mathrm{~nm}$ (the width of the GNRs), and $\mathrm{d}=5 \mathrm{~nm}$ (the coupling distances between the GNRs and the GSNR). The coupling distance, $\mathrm{d}$, is chosen so that it guarantees an effective coupling of the surface plasmon polariton (SPP) waves between the components [37, 38]. The width of the GNRs is fixed through all of the simulations to assure of the edge mode propagation only and the potential application of the structure in the ultra-compact integrated devices. The structures are shown in Fig. 1(a)-Fig. 1(c) is designed to be patterned on a sapphire film which is deposited on the silicon substrate. The $\mathrm{Si} / \mathrm{Al}_{2} \mathrm{O}_{3}$ substrate is chosen because it has strong absorption in MIR and low loss propagation. Therefore, it is the best candidate to support GNRs instead of $\mathrm{SiO}_{2}$. The refractive index and extinction coefficient $k$ of $\mathrm{Al}_{2} \mathrm{O}_{3}$ (Palik) are 1.59 and 0.0020 at $6 \mu \mathrm{m}$, respectively [39]. Also, in all the simulation runs, the thickness of the substrate is selected to be $50 \mathrm{~nm}$ for $\mathrm{Al}_{2} \mathrm{O}_{3}$ and $500 \mathrm{~nm}$ for $\mathrm{Si}$.

The design, dimensions, and materials of the proposed structures have been chosen to meet the fabrication considerations. The development of the proposed structure is technically feasible with well-developed patterning methods that have recently been used to produce GNRs with similar geometrical size features [40-42]. In a typical fabrication process, a highquality large-area graphene layer can be grown on a copper substrate. After the etching process of $\mathrm{Cu}$, it is floated on the deionized water and then is pulled out on the substrate. Using electron-beam lithography and oxygen plasma, the GNRs can be patterned and the device is formed [43, 44]. It is worth mentioning that for the proposed structures, the graphene monolayer is considered to be transferred to the $\mathrm{Si} / \mathrm{Al}_{2} \mathrm{O}_{3}$ substrate. 


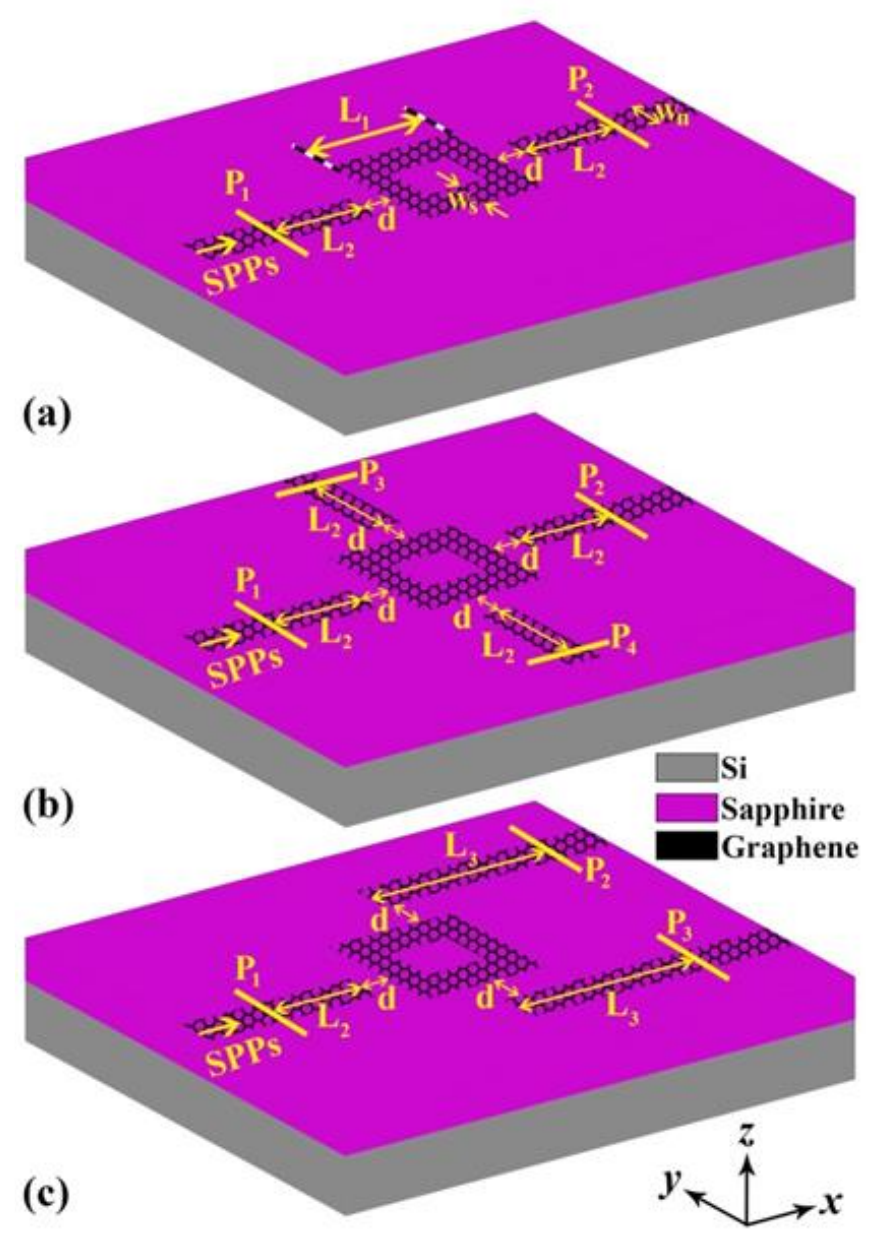

Figure 1. Three-Dimensional (3D) schematic diagram of the proposed structures based on GSNR for different applications (a) Band-pass filter. (b) Wavelength demultiplexer. (c) Power splitter.

Graphene, as an ultra-thin film with a thickness of $\Delta \approx 0.34 \mathrm{~nm}$, guides the surface plasmonic waves in the MIR region. The surface conductivity of a graphene single layer is yielded from the well-known Kubo formula [30, 45, 46]:

$$
\sigma_{g}=i \frac{e^{2} k_{B} T}{\pi \eta^{2}\left(\omega+i \tau^{-1}\right)}\left[\frac{\mu_{c}}{k_{B} T}+2 \ln \left(\exp \left(-\frac{\mu_{c}}{k_{B} T}\right)+1\right)\right]+i \frac{e^{2}}{4 \pi \eta^{2}} \ln \left[\frac{2\left|\mu_{c}\right|-\eta\left(\omega+i \tau^{-1}\right)}{2\left|\mu_{c}\right|+\eta\left(\omega+i \tau^{-1}\right)}\right]
$$

Where $\omega, \mu_{\mathrm{c}}, \hbar, T, k_{\mathrm{B}}, e$, and $\tau$ are the angular frequency of the electromagnetic wave, chemical potential of the graphene, the reduced Plank's constant, the temperature, the Boltzmann's constant, the electron charge, and the carriers relaxation time of the material, respectively. Two terms are contained in the Kubo formula including the intra-band (the first term in Eq.1) and the inter-band (the second term) transition contributions. In the MIR wavelengths, which is subjected working region in this article, the energy of photons, $E_{\mathrm{ph}}$, is low enough which let the $\mu_{c}$ to be always above half of it (i.e., $\hbar \omega<<2 \mu_{c}$ ), then the inter-band transition is ignored here, and the $\sigma_{\mathrm{g}}$ is reduced to the semi-classical Drude model [30, 47]: 


$$
\sigma_{g}=i \frac{e^{2} \mu_{c}}{\pi \eta^{2}\left(\omega+i \tau^{-1}\right)}
$$

Eq. 2 determines the absolute value of the graphene chemical potential. The momentum relaxation time depends on the DC mobility $(\mu)$, the $\mu_{c}$, and the Fermi velocity $\left(v_{\mathrm{F}} \approx 10^{6} \mathrm{~m} / \mathrm{s}\right)$;

$$
\tau=\mu \mu_{c} /\left(e v_{F}^{2}\right)
$$

Through the simulations, the single-layer graphene is modeled as an anisotropic dielectric, with the in-plane permittivity. If $\varepsilon_{0}$ is to be the vacuum permittivity, then the effective permittivity of the material is expressed as Eq. $4[30,47]$.

$$
\varepsilon_{g}=1+i \frac{\sigma_{g}}{\omega \varepsilon_{0} \Delta}
$$

The resonance wavelength of the GSNR, which works as the resonator through all the simulations, is $[24,30]$ :

$$
\lambda=\frac{4 L_{e f f} \operatorname{Re}\left(n_{e f f}\right)}{m}, m=1,2,3 \ldots
$$

Here, the integer $m$ is the order of the resonance mode and $L_{\text {eff }}$ is the effective side length of the resonator, which is the mean of the internal and external sides of the GSNR resonator. In the analysis of the performance of the proposed structures, a key parameter is the $n_{\text {eff }}$ a demonstration of the effective refractive indices in the SPPs modes which are supported by the GSNR. $n_{\text {eff }}$ is affected by several parameters within the models which are explained in the next section, and it can be obtained from the dispersion equation with the $\eta_{0}(\approx 377 \Omega)$ as the impedance of the air [48]:

$$
n_{\text {eff }}=\sqrt{1-\left(\frac{2}{\sigma_{g} \eta_{0}}\right)^{2}}
$$

Therefore, the propagation constant of the SPPs in the GSNR is $\beta_{\mathrm{SPP}}=k_{0} n_{\mathrm{eff}}$, where $k_{0}$ is the free space wave vector.

The models are simulated using 3D-FDTD in Lumerical FDTD Software package with 24 identical perfectly matched layers as the absorbing boundary conditions all around the numerical simulation zone. The dipole point source, $2 \mathrm{~nm}$ over the location of the input monitor $\left(\mathrm{P}_{1}\right)$, was used to excite the incident SPP waves with transverse magnetic (TM) polarization and the power of $\mathrm{P}_{\mathrm{in}}$, in the $+x$ direction. The output monitors $\left(\mathrm{P}_{2}-\mathrm{P}_{4}\right)$ are set at the end of the output GNR waveguides to detect the transmitted powers $\mathrm{P}_{\text {out }}$. The normalized transmittance is then calculated as $\mathrm{T}=\mathrm{P}_{\text {out }} / \mathrm{P}_{\text {in }}$. As long as edge modes are activated, the 
orientation of the dipole has an insignificant impact on the results. To obtain reliable, accurate, and at the same time, time-effective solutions, the numerical calculations used nonuniform mesh grid sizes of $0.1 \mathrm{~nm}$ for graphene, in the $z$-direction, while in the vicinity of the graphene components, the mesh size was $1 \mathrm{~nm} \times 1 \mathrm{~nm}$ in the $x-y$ plane.

\section{Results and Discussions}

In this section, the functions of different structures presented in Fig. 1 are introduced and analyzed, such as the filter, demultiplexer, and power splitter. In all simulations, the $\mu_{\mathrm{c}}$ for GNR waveguides is assumed to be constant and equal to $0.4 \mathrm{eV}$, and the $\mu_{\mathrm{c}}$ for the GSNR is flexibly adjusted by controlling different gate voltages applied to graphene.

\subsection{Filter}

To analyze the performance of the plasmonic band-pass filter (BPF), the structure shown in Fig. 1(a) is proposed. This structure consists of a GSNR and two GNR waveguides as input/output waveguides. The dipole point source excites the SPP waves at the edge of the input GNR waveguide (edge mode) and they are then coupled to the GSNR resonator. These waves are back and forward waves at the edge of the input GNR waveguide as well as the GSNR resonator, causing standing waves in the GSNR (here GSNR acts like the Fabry-Perot resonator (FPR)). As a result, the input wavelengths which satisfy the GSNR resonant conditions (Eq. (4)) can be effectively coupled to the output GNR and the other wavelengths are stopped. In Fig. 1(a), the two monitors are positioned $\mathrm{P}_{1}$ and $\mathrm{P}_{2}$, respectively, to detect incident power and transmitted power, and the transmittance is defined as $\mathrm{T}=\mathrm{P}_{2} / \mathrm{P}_{1}$.

The transmission spectrum of the structure is shown in Fig. 2(a). The geometric parameters of the structure are equal to $\mathrm{L}_{1}=\mathrm{L}_{2}=100 \mathrm{~nm}, \mathrm{~W}_{\mathrm{s}}=\mathrm{W}_{\mathrm{n}}=20 \mathrm{~nm}$, and d=5 nm. The $\mu_{\mathrm{c}}$ for GNR waveguides and GSNR is considered constant at $0.4 \mathrm{eV}$. Three transmission peaks in the wavelength range of 6.5 to $11 \mu \mathrm{m}$ pass through the structure, which corresponds to the resonant modes of the $4^{\text {th }}$ order $\left(\lambda_{4}=7.39 \mu \mathrm{m}\right)$ and the $3^{\text {rd }}$ order $\left(\lambda_{3}=8.26 \mu \mathrm{m}\right)$, and the $2^{\text {nd }}$ order $\left(\lambda_{2}=10.11 \mu \mathrm{m}\right)$, respectively. These peaks have transmission amplitude of $0.44,0.54$, and 0.58 , respectively. To evaluate the performance of the BPF, we use the quality factor, which is defined as $\mathrm{Q}=\lambda_{0} / \Delta \lambda$, where $\lambda_{0}$ and $\Delta \lambda$ are the central wavelength and full width at half maximum (FWHM), respectively. Table 1 shows the specifications of the different orders of resonance of the designed structure. As shown in Table 1, the quality factor decreases with an increasing central wavelength of the resonant mode. Therefore, there is a trade-off between these two parameters. 
Table 1: Characteristics of different orders of resonant modes.

\begin{tabular}{cccc}
\hline Order Mode & $\lambda_{0}(\mu \mathrm{m})$ & FWHM $(\Delta \lambda)(\mu \mathrm{m})$ & Quality Factor \\
\hline $4^{\text {th }}$ order mode & 7.39 & 0.18 & 41.05 \\
$3^{\text {rd }}$ order mode & 8.26 & 0.32 & 25.81 \\
$2^{\text {nd }}$ order mode & 10.11 & 0.47 & 21.51 \\
\hline
\end{tabular}

Table 2 shows the comparison between the maximum transmission ratio in this work with other graphene-based structures, which compared to other structures, the proposed structure has a larger maximum transmission ratio.

Table 2: Comparison of maximum transmission ratio in different works.

\begin{tabular}{ccccccc}
\hline Reference & {$[49]$} & {$[50]$} & {$[51]$} & {$[24]$} & {$[52]$} & This work \\
\hline $\begin{array}{c}\text { Maximum } \\
\text { Transmission Ratio }\end{array}$ & 0.25 & 0.38 & 0.4 & 0.45 & 0.5 & 0.58 \\
\hline
\end{tabular}

Figures 2(b)-2(d) show the spatial distribution profiles of $H_{z}$ from the cross-sectional view $x y$ for the wavelengths corresponding to the fourth, third, and second-order resonant modes, respectively. The GSNR resonator also acts as an FPR cavity.
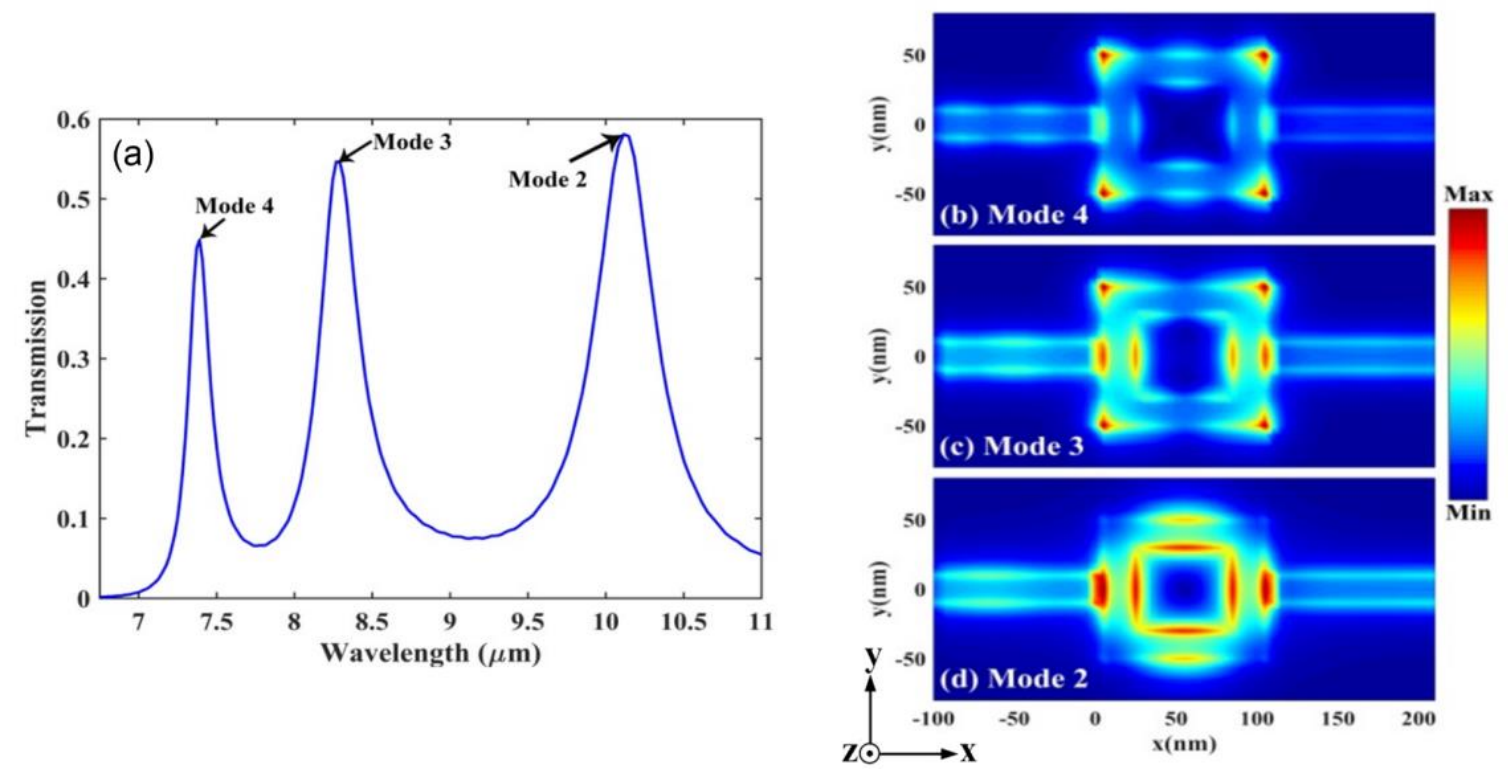

Figure 2. (a) The transmission spectrum of the proposed BPF structure is shown in Fig. 1(a). (b)-(d) Spatial distributions of $H_{z}$ at the peaks of transmission for resonance modes.

The effect of different parameters such as $\mu_{\mathrm{c}}$, side lengths of GSNR ( $\left.\mathrm{L}_{1}\right)$, coupling distance between input/output GNR waveguides with GSNR (d), and width of GSNR $\left(\mathrm{W}_{\mathrm{s}}\right)$ on the filter transmission spectrum is shown in Fig. 3. 
The transmission spectra calculated for the different values of the $\mu_{\mathrm{c}}$ of the GSNR obtained from applying the external gate voltage are shown in Fig. 3(a). The other parameters are set as shown in Fig. 2. As can be seen from Fig. 3 (a), the resonance wavelength of the GSNR decreases with increasing $\mu_{\mathrm{c}}$ and the transmission spectrum experiences a blueshift. According to Eqs. (2), (5), and (6), this blueshift can be explained because increasing $\mu_{\mathrm{c}}$ leads to a reduction in $n_{\text {eff }}$ and thus a decrease in the resonance wavelength. Moreover, with increasing $\mu_{\mathrm{c}}$, transmittance power increases. Figure 3 (b) shows the effect of changing the $\mathrm{L}_{1}$ on the transmission spectrum. It is clear that by increasing $\mathrm{L}_{1}$ for a constant $\mu_{\mathrm{c}}(0.4 \mathrm{eV})$, the transmission peaks undergo redshift, which is resulted in Eq. (5). Besides, the coupling distance (d) between the input/output GNR waveguides and the GSNR affects the transmission peaks and the resonance wavelength, as shown in Fig. 3 (c). In this figure, by increasing the parameter $\mathrm{d}$, besides the transmission spectrum experiencing a small blueshift resulting from the phase shift of the SPPs, the transmission peak and the stop-band level also decreases. Find that due to the weakening of the coupling distance between the waveguide and the resonator. Furthermore, the transmission spectrum of the $\mathrm{W}_{\mathrm{s}}$ is investigated in Fig. 3 (d). As the $\mathrm{W}_{\mathrm{s}}$ increases from 15 to $20 \mathrm{~nm}$, the transmission spectrum has a blueshift because the $n_{\text {eff }}$ of the GSNR decreases, and according to Eq. (4), the resonance wavelength decreases. It is clear from the simulation results that the structure presented in Fig. 1(a) can be used as a plasmonic BPF with dynamic tunability without re-fabrication of the structure in the MIR spectral region. On the other hand, the proposed structure can be utilized in plasmonic devices due to its simplicity in terms of fabrication. 

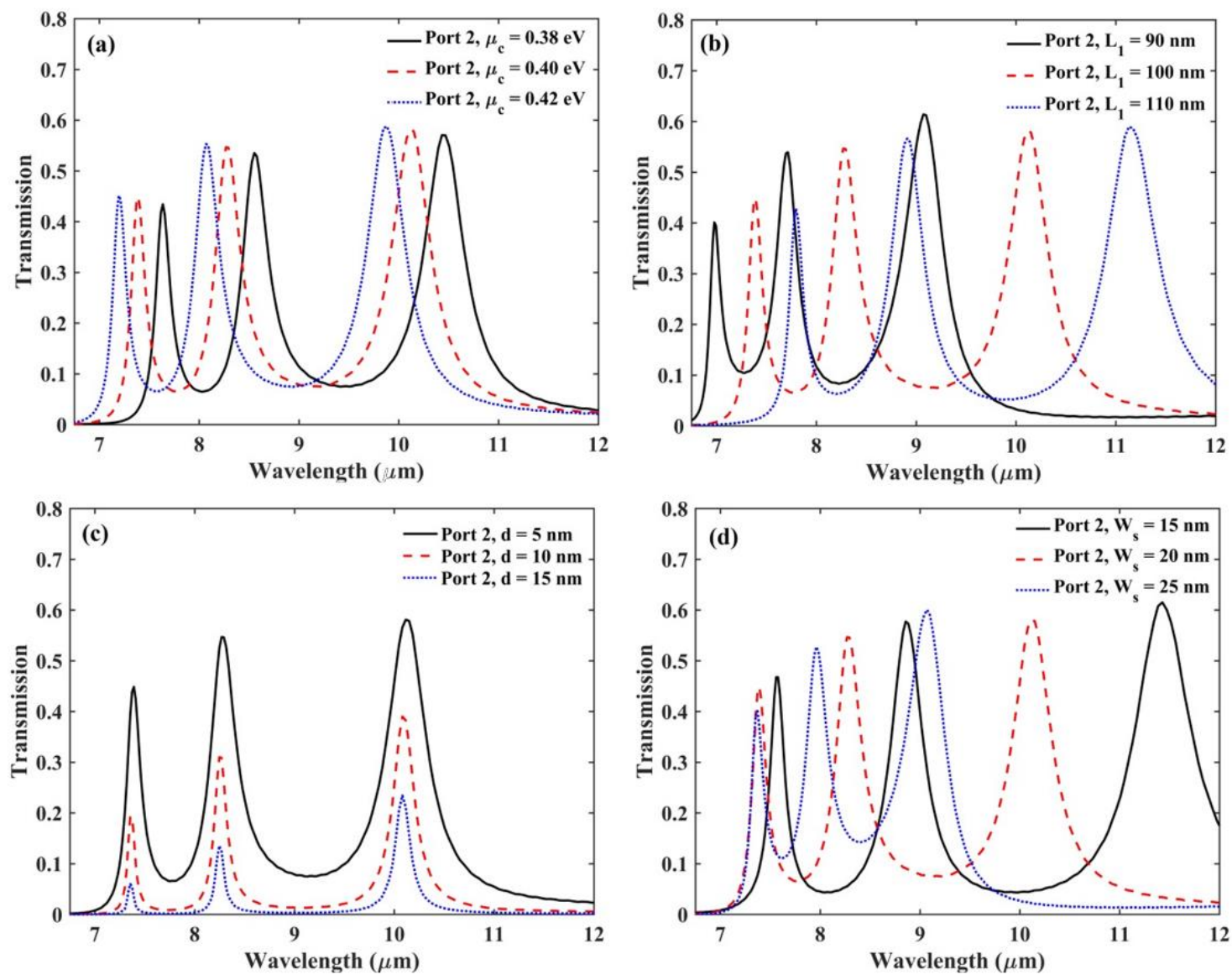

Figure 3. The transmission spectra of the proposed BPF structure are shown in Fig. 1(a) for different (a) $\mu_{\mathrm{c}}$, (b) side lengths $\left(\mathrm{L}_{1}\right)$ of GSNR, (c) coupling distance (d) between input/output GNR waveguides and GSNR, and $(\mathrm{d})$ width $\left(\mathrm{W}_{\mathrm{s}}\right)$ of GSNR.

\subsection{Wavelength Demultiplexer}

Figure 1(b) shows the structure of a plasmonic demultiplexer consisting of a GSNR which is performed as a resonator and four GNR waveguides as input/output waveguides which are symmetrically placed around the GSNR. The geometric parameters of the structure are similar to structure Fig. 1(a) and since the proposed structure is symmetrical, only port 1 which is set as input, and ports 2 and 3 which are set as two outputs (output port 3 has the same position as port 4) are examined as follows and the results are the same for the two output ports 3 and 4 due to symmetry. To reveal the incident power and transmitted power, three monitors are used in positions $\mathrm{P}_{1}, \mathrm{P}_{2}, \mathrm{P}_{3}$, respectively, and the transmittance is defined as $\mathrm{T}=\mathrm{P}_{2} / \mathrm{P}_{1}$ and $\mathrm{T}=\mathrm{P}_{3} / \mathrm{P}_{1}$, and the distance between these three monitors and the GSNR is equal to $\mathrm{L}_{2}=100 \mathrm{~nm}$.

The demultiplexer transmission spectra with the parameters $\mu_{\mathrm{c}}=0.4 \mathrm{eV}, \mathrm{L}_{1}=\mathrm{L}_{2}=100 \mathrm{~nm}$, $\mathrm{W}_{\mathrm{s}}=\mathrm{W}_{\mathrm{n}}=20 \mathrm{~nm}$, and $\mathrm{d}=5 \mathrm{~nm}$ are shown in Fig. 4(a). In transmission spectra, graphene SPP 
waves can only be transmitted through the output port 2 during the $3^{\text {rd }}$ resonance wave $(\lambda=$ $8.29 \mu \mathrm{m})$ and have a high transmission amplitude of 0.56 , while the transmission from through output port 3 (vertical port) is negligible and equal to 0.01 . This result completely agrees with the rule of the signal intersection at the resonance wavelength (port $1 \leftarrow \rightarrow$ port 2 and port $3 \leftarrow \rightarrow$ port 4$)$. For the $2^{\text {nd }}$ resonance wavelength $(\lambda=10.18 \mu \mathrm{m})$ and the $4^{\text {th }}$ resonance wavelength $(\lambda=74.74 \mu \mathrm{m})$, the amplitudes of the transmission for the two output ports are approximately equal to 0.18 and 0.15 , respectively. Hence, the structure is simultaneously served as two device consist of a demultiplexer ( $3^{\text {rd }}$ resonance wavelength), and a tunable $1 \times 3$ coupler $\left(2^{\text {nd }}\right.$ and $4^{\text {th }}$ resonance wavelengths) with constant splitting ratio $(1: 1: 1)$ or $1 \times 2$ coupler when port 4 is closed. For better understanding, the transmission mechanism of the proposed structure, the spatial distribution profile of $H_{\mathrm{z}}$ for the wavelengths corresponding to the $4^{\text {th }}, 3^{\text {rd }}$, and $2^{\text {ed }}$ order resonant modes are depicted in Figs. 4(b)-4(d), respectively. As shown in Figs. 4(b) and 4(d), the edge modes are excited and propagated evenly along with the output of the GNR waveguides, and easily form a tunable $1 \times 3$ coupler. It is also clear from Fig. 4 (c) that for output port 2, the resonant mode is located in the GSNR resonator and the exciting edge mode and power pass-through output port 2, and almost no power cannot be coupled to output ports 3 and 4 .

Moreover, the demultiplexer performance of the structure can be evaluated by two important factors, namely, insertion loss (IL) and extinction ratio (ER). These two factors are defined as $\mathrm{IL}=10 \log \left(\mathrm{P}_{w} / \mathrm{P}_{i}\right)$ and $\mathrm{ER}=10 \log \left(\mathrm{P}_{w} / \mathrm{P}_{u w}\right)$, respectively [53], in which $\mathrm{P}_{i}$ represents the power at the input waveguide and $\mathrm{P}_{w}$ and $\mathrm{P}_{u w}$ are related to the desired and undesirable optical powers in the output waveguides. For the $3^{\text {rd }}$ resonance wavelength $(\lambda=$ $8.29 \mu \mathrm{m})$, the output port 2 and the output port 3 represent the desired $\left(\mathrm{P}_{w}\right)$ and undesirable $\left(\mathrm{P}_{u w}\right)$ output powers, respectively. Therefore, by applying the above equation, IL and ER for the $3^{\text {rd }}$ resonance wavelength are equal to $2.5 \mathrm{~dB}$ and $16.55 \mathrm{~dB}$, respectively. 

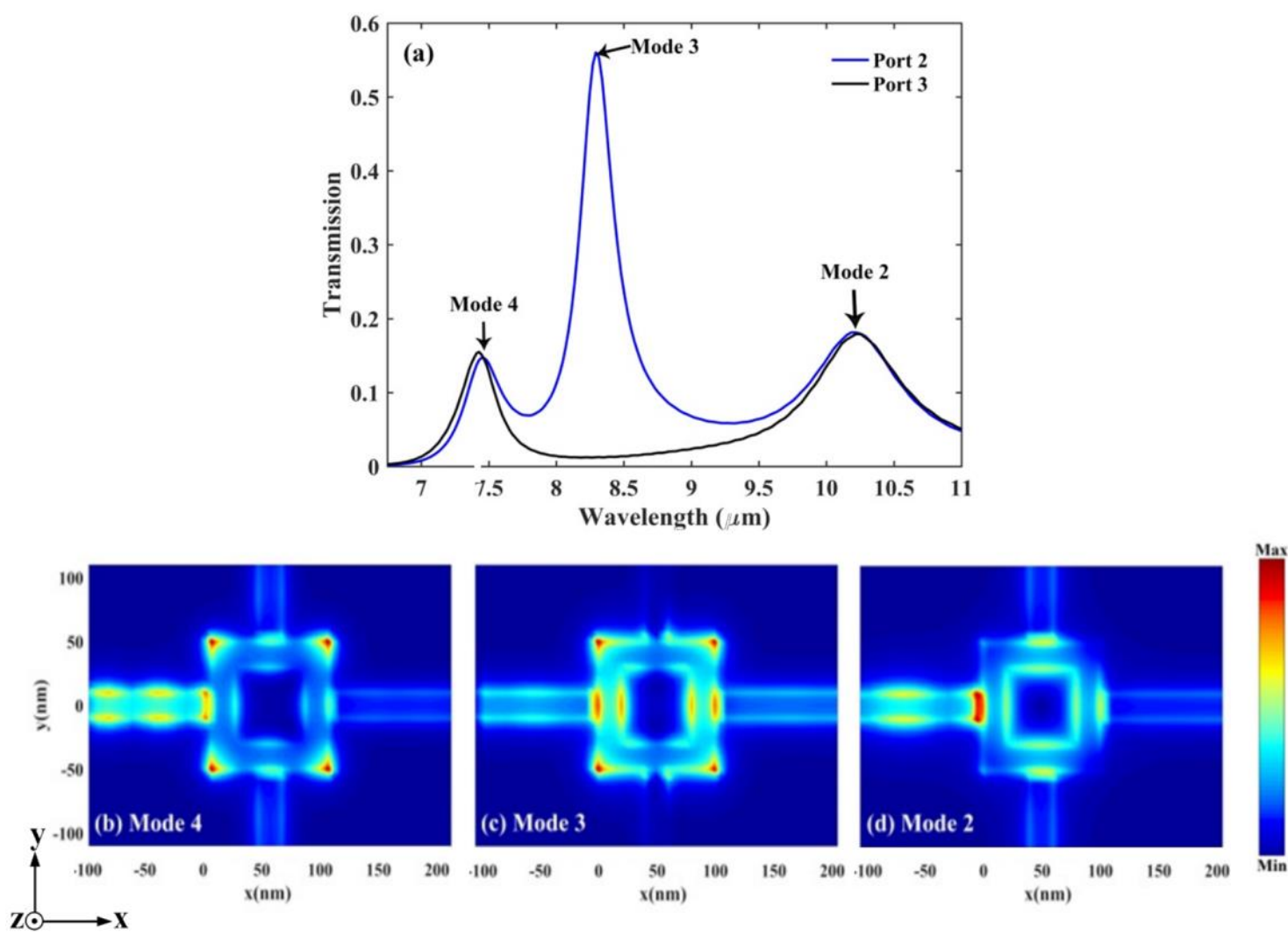

Figure 4. (a) The transmission spectra of ports 2 and 3 of the proposed wavelength demultiplexer structure are shown in Fig. 1(b). (b)-(d) The spatial distributions of $H_{z}$ at the peaks of transmission for resonance modes.

The performance of various parameters in this structure is shown in Fig. 5. The demultiplexer transmission spectrum for different $\mu_{\mathrm{c}}$ from 0.38 to $0.42 \mathrm{eV}$ with 0.02 steps with geometric parameters $\mathrm{L}_{1}=\mathrm{L}_{2}=100 \mathrm{~nm}, \mathrm{~W}_{\mathrm{s}}=\mathrm{W}_{\mathrm{n}}=20 \mathrm{~nm}$, and $\mathrm{d}=5 \mathrm{~nm}$ is illustrated in Fig. 5(a). As obvious in Fig. 5 (a), as the $\mu_{\mathrm{c}}$ increases, the transmission spectrum experiences a blueshift, as explained in the previous section. The effect of GSNR dimensions on the transmission spectrum and its results are shown in Fig. 5(b). Other parameters are constant and equal to $\mu_{\mathrm{c}}=0.4 \mathrm{eV}, \mathrm{W}_{\mathrm{s}}=\mathrm{W}_{\mathrm{n}}=20 \mathrm{~nm}$, and $\mathrm{d}=5 \mathrm{~nm}$. As it is known, by increasing the dimensions of the GSNR, the transmission spectrum undergoes a redshift, which can be explained according to the GSNR conditions (Eq. (4)) since the GSNR dimensions increases, the resonance wavelength increases. Another important parameter effect, the different coupling distance between the input/output GNR waveguides and the GSNR, on the transmission spectrum of the two output ports 2 and 3 is shown in Fig. 5(c). The $\mu_{\mathrm{c}}$ for GSNR and GNR waveguides is the same and is equal to $0.4 \mathrm{eV}$. As can be seen from this figure, the coupling length between the GNR waveguides and the GSNR has a low effect on the resonance wavelength; because the resonant mode changes only with the geometric 
parameters and $\mu_{\mathrm{c}}$ of the GSNR (transmission spectrum has a negligible blueshift due to SPP phase shift). It is also clear that as the coupling length increases, the coupling loss increase, and thus the transmission amplitude decreases. To have ultra-compact devices with a high ER, the width of the GNR input/output waveguides and the GSNR must be chosen so that the dimensions of the device are compressed and reduced. Therefore, in all structures, the width of the GNR and GSNR is less than the critical values to be able to excite only the edge mode. However, the effect of different $\mathrm{W}_{\mathrm{s}}$ on the transmission spectrum of the two output ports 2 and 3 is shown in Fig. 5(d). By increasing the $\mathrm{W}_{\mathrm{s}}$ from 15 to $25 \mathrm{~nm}$, the transmission spectrum of the two output ports, in addition to increasing its amplitude, also experiences a redshift. This behavior can be understated by using Eq. (4) and the same as the previous section.
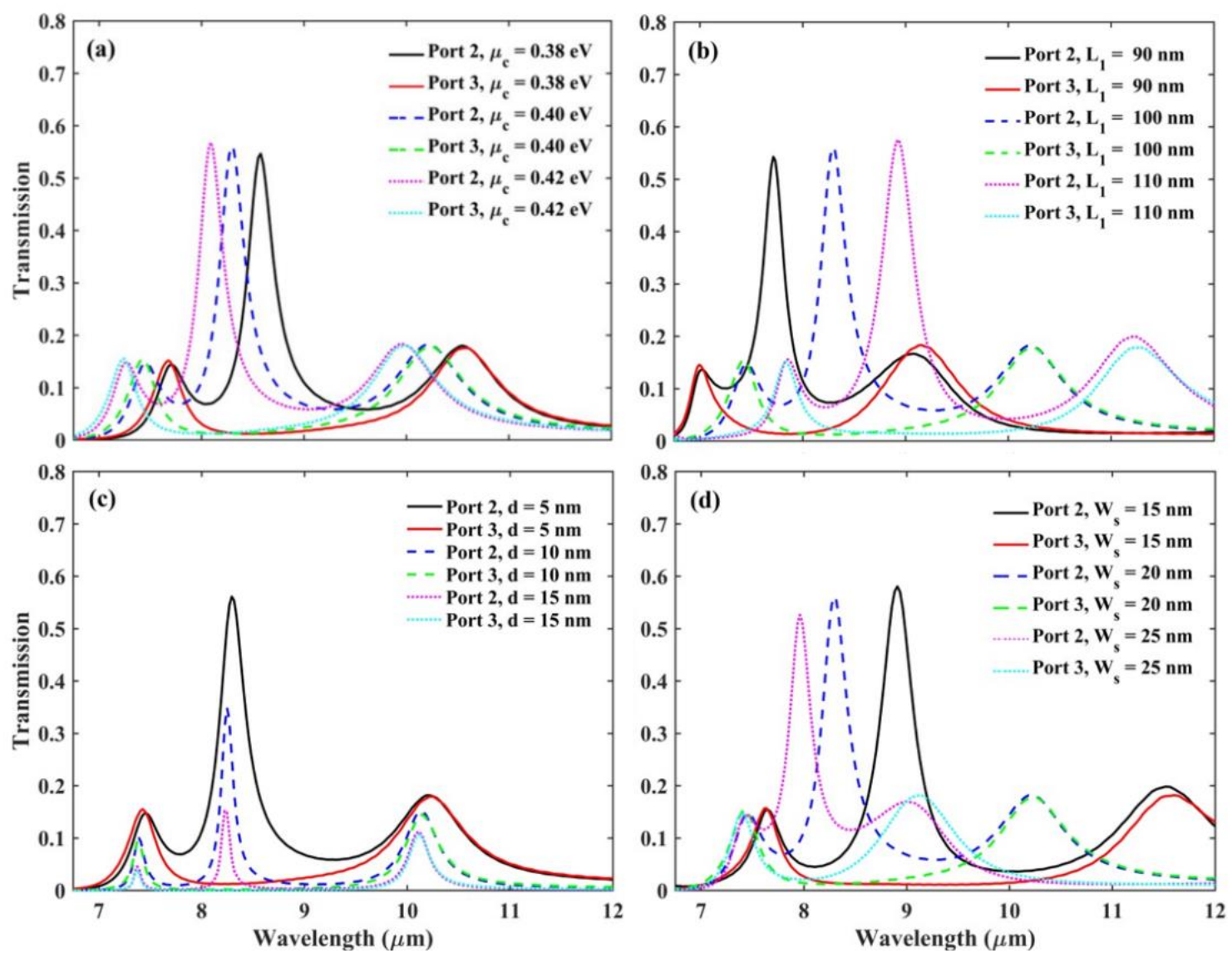

Figure 5. The transmission spectra of ports 2 and 3 of the proposed wavelength demultiplexer structure are shown in Fig. 1(b) for different (a) $\mu_{\mathrm{c}}$, (b) side lengths $\left(\mathrm{L}_{1}\right)$ of GSNR, (c) coupling distance (d) between input/output GNR waveguides and GSNR, and (d) width $\left(\mathrm{W}_{\mathrm{s}}\right)$ of GSNR. 

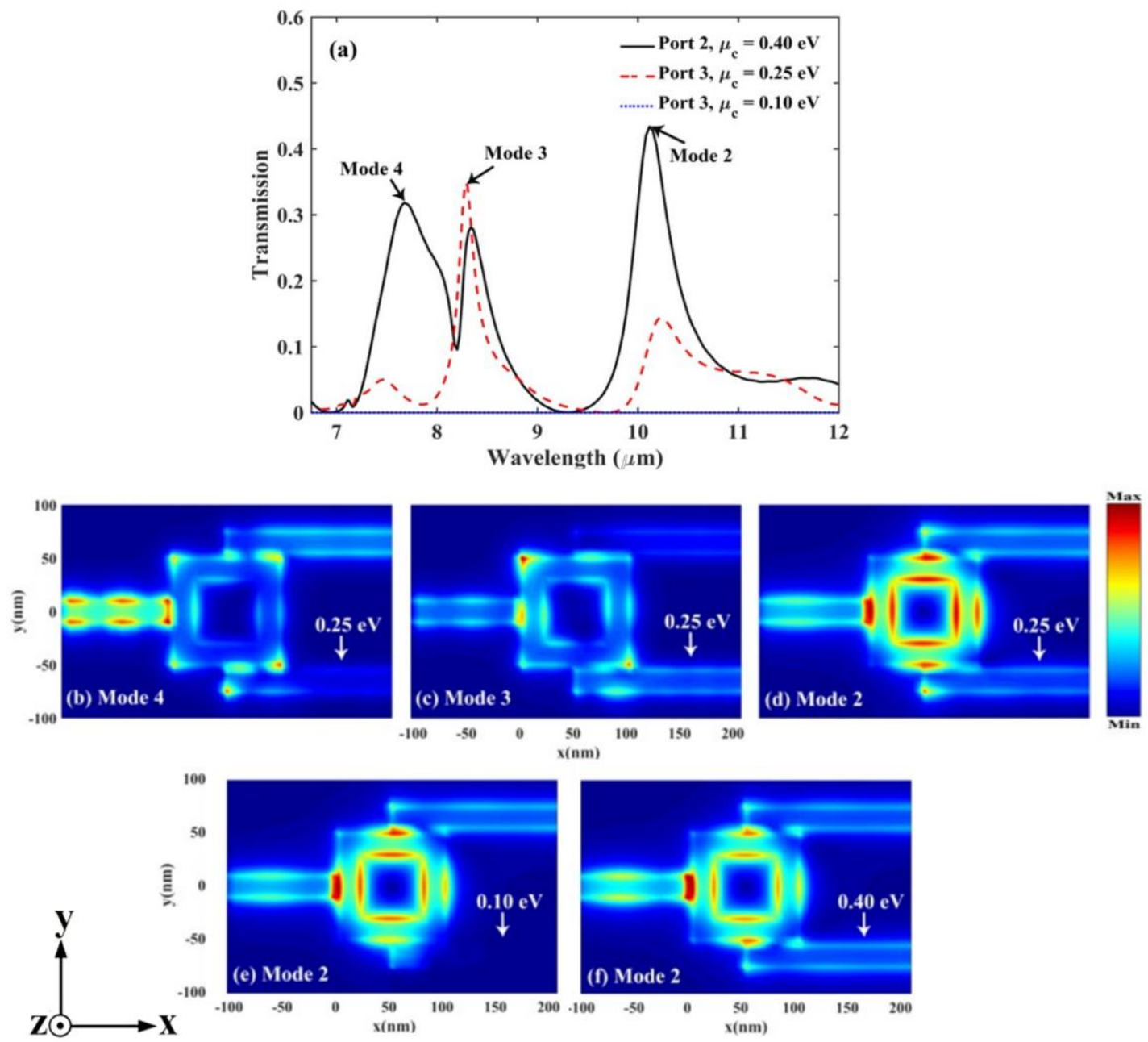

Figure 6. (a) The transmission spectra of ports 2 and 3 of the proposed power splitter structure are shown in Fig. 1(c) for different $\mu_{\mathrm{c}}$ for port 3 and $\mu_{\mathrm{c}}=0.40 \mathrm{eV}$ for port 2. (b)-(d) the spatial distributions of $H_{z}$ for resonance modes with $\mu_{\mathrm{c}}=0.25 \mathrm{eV}$ for port 3. (e) Spatial distribution of $H_{z}$ for the mode 2 with $\mu_{\mathrm{c}}=0.10 \mathrm{eV}$ for port 3. (f) Spatial distribution of $H_{z}$ for the mode 2 with $\mu_{\mathrm{c}}=0.40 \mathrm{eV}$ for both ports.

\subsection{Power Splitter}

To employ the effective coupling between the output GNR waveguides and the GSNR, two GNR waveguides are added in parallel at the top and bottom of the GSNR, as shown in Fig. 1(c). By adding these two GNR waveguides to the output, the input energy can have two practical waveguides. This structure can be used as a power splitter at some resonance wavelengths. The power splitter is one of the basic functional elements in nano-plasmonic integrated devices and circuits. In Fig. 1(c), the monitors are in position $\mathrm{P}_{1}$ to detect incident power and the two monitors are in positions $\mathrm{P}_{2}$ and $\mathrm{P}_{3}$ to detect transmitted power, and the transmittance is defined as $\mathrm{T}=\mathrm{P}_{2} / \mathrm{P}_{1}$ and $\mathrm{T}=\mathrm{P}_{3} / \mathrm{P}_{1}$. Here, only the effect of two parameters, namely, $\mu_{\mathrm{c}}$ and coupling distance (d) between two output GNR waveguides with GSNR is 
investigated and the results are given as follows. The other parameters are fixed structures similar to the previous two structures.

By applying the gate voltage and its effect on the $\mu_{\mathrm{c}}$ at the two output ports, the power splitting ratio can be altered and thus the transmission spectrum changed at the two output ports, as is shown in Fig. 6(a). Here, the $\mu_{\mathrm{c}}$ of the output port 2 is fixed and equated to $\mu_{\mathrm{c}}=$ $0.40 \mathrm{eV}$ and the $\mu_{\mathrm{c}}$ of the output port 3 is set once $\mu_{\mathrm{c}}=0.25 \mathrm{eV}$ and again $\mu_{\mathrm{c}}=0.10 \mathrm{eV}$. When the $\mu_{\mathrm{c}}$ of output ports 2 and 3 is set to 0.40 and 0.25 , respectively, for the $2^{\text {nd }}$ resonance wavelength $(\lambda=10.15 \mu \mathrm{m})$, the transmission amplitude for output port 2 is 0.42 and for the output port 3 is 0.12 . The spatial distribution profile of $H_{z}$ for this case is shown in Fig. 6(d). In the $3^{\text {rd }}$ resonance wavelength $(\lambda=8.29 \mu \mathrm{m})$, the transmission for the output port 3 is 0.34 and for the output port 2 is 0.25 , and the spatial distribution profile of $H_{\mathrm{z}}$ is given in Fig. 6(c). In these two resonance wavelengths, the structure acts as a power splitter that has a different splitter ratio. These results can be justified using the graphene conductivity equation (Eq. (2)) because increasing the $\mu_{\mathrm{c}}$ leads to increasing the $\sigma_{\mathrm{g}}$. Therefore, the transmission loss along with the GNR decrease and the transmission amplitude at the output ports increases with increasing $\mu_{\mathrm{c}}$. In contrast to the above discussion, when the wavelength is $8.29 \mu \mathrm{m}$, the transmission at output port 3 is slightly more than the transmission at output port 2 . Since the coupling between output waveguide 2 and GSNR is slightly weaker than output port 3 . Therefore, the input signal beam is coupled directly to the output port 3 . For the $4^{\text {th }}$ resonance wavelength $(\lambda=7.67 \mu \mathrm{m})$, the value of the transmission amplitude for the output port 2 is 0.31 , while the value for the output port 3 is sharply reduced to nearly zero (0.02). Therefore, the proposed structure acts as a demultiplexer. The spatial distribution profile of $H_{z}$ for this case is shown in Fig. 6(b). When the $\mu_{\mathrm{c}}$ of output port 3 is adjusted to $0.10 \mathrm{eV}$, the SPP transmission loss in the output port 3 is greatly increased, the SPP waves are reflected, and it cannot support any graphene surface plasmon in the wavelength range of 6 to $12 \mu \mathrm{m}$. The spatial profile of $H_{\mathrm{z}}$ in Fig. 6(e) confirms this issue. The spatial profile of $H_{\mathrm{z}}$ for when the $\mu_{\mathrm{c}}$ of both output ports is equal to $\mu_{\mathrm{c} 2}=\mu_{\mathrm{c} 3}=0.40 \mathrm{eV}$, is shown in Fig. 6(f), and the transmission amplitude both ports are the same and equal to 0.42 .

The transmission spectrum of the power splitter structure in Fig. 1(c) strongly depends on the coupling distance $(d)$ between the two output GNR waveguides and the GSNR. The strong optical coupling between the output GNR waveguides and the GSNR requires a small mismatch in the propagation constant of the SPPs along with the GNR, which satisfies the phase-matching conditions. The coupling ability between the output GNR waveguides and 
the GSNR can be managed by changing the $d$, as shown in Fig. 7(a). In this figure, the transmission spectrum is shown for a situation where the coupling distance between the GSNR and output port 2 and port 3 is $5 \mathrm{~nm}$ and $10 \mathrm{~nm}$, respectively. For the $3^{\text {rd }}$ wavelength $(\lambda=8.31 \mu \mathrm{m})$, the transmission for both output ports is 0.21 , and the proposed structure operates similar to a $1 \times 2$ power splitter. The spatial distribution profile of $H_{\mathrm{z}}$ for this case is shown in Fig. 6(c). For the $2^{\text {nd }}$ resonance wavelengths $(\lambda=10.11 \mu \mathrm{m})$ and the $4^{\text {th }}$ resonance wavelengths $(\lambda=7.44 \mu \mathrm{m})$, the structure acts as a demultiplexer. At these resonance wavelengths, the transmission amplitude for output port 2 is 0.44 and 0.33 , respectively, while the transmission amplitude for output port 3 is strongly suppressed and is nearly zero. The spatial distribution profile of $H_{\mathrm{z}}$ for these two resonance wavelengths is given in Fig. 6(b) and 6(d). This figure shows that as the length of the coupling increases, the intensity of the coupling to the output port decreases dramatically.
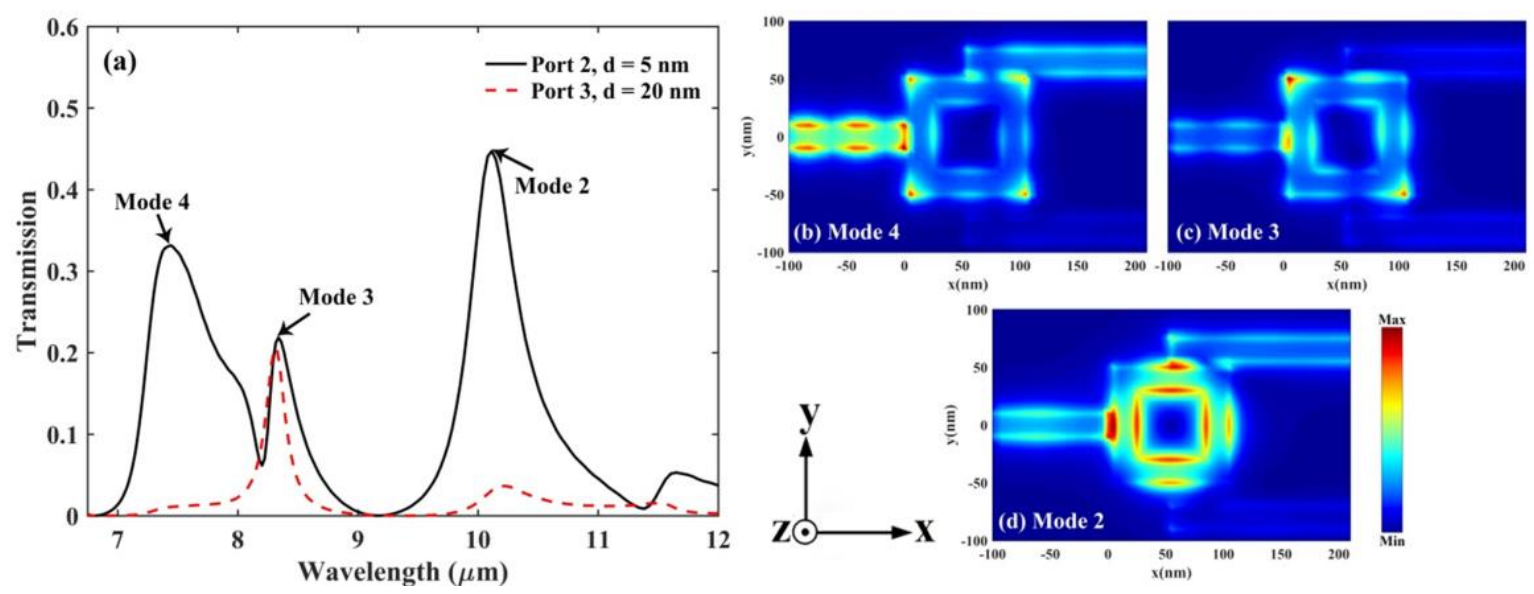

Figure 7. (a) The transmission spectra of ports 2 and 3 of the proposed power splitter structure are shown in Fig. 1(c) for different d. (b)-(d) The spatial distributions of $H_{z}$ at the peaks of transmission for resonance modes.

\section{Conclusion}

In summary, three fundamental devices including a filter, a demultiplexer, and a power splitter were designed for application in nanophotonic integrated circuits. The abovementioned arrangements were theoretically and numerically analyzed using the 3DFDTD method. These structures consisted of the main block, i.e. a GSNR, as a resonator and a set of some extra GNR waveguides as input/outputs. The structures were very convenient and simple in terms of implementation and practical applications. The coupling intensity of GSPPs between GSNR and GNR output waveguides is affected by various parameters such as chemical potential, coupling length, and dimensions of GSNR, and so does the 
transmission spectrum at the output of waveguides, as a consequence. Changing these parameters the coupling intensities could be controlled and tuned in the MIR spectral range.

\section{Declarations}

Funding This article has no funding.

Conflicts of Interest The authors declare no competing interests.

Availability of Data and Material All datasets generated and/or analyzed during this study are available from the corresponding author on reasonable request.

Code Availability The code used during the current study is available from the corresponding author on reasonable request.

Authors' Contributions Design, Methodology and numerical simulations of the paper: Morteza Janfaza. Writing of the manuscript: Morteza Janfaza. Writing-comments and suggestions: Mohammad Ali Mansouri-Birjandi and Alireza Tavousi.

Ethics Approval The authors have followed the ethical principles and accurate references to scientific sources in the original paper.

Consent to Participate All authors have contributed to this article.

Consent for Publication All authors agree to publish this article. 


\section{References}

[1] W. L. Barnes, A. Dereux, and T. W. Ebbesen, "Surface plasmon subwavelength optics," Nature, vol. 424, no. 6950, pp. 824-830, 2003.

[2] W. Srituravanich, N. Fang, C. Sun, Q. Luo, and X. Zhang, "Plasmonic nanolithography," Nano Lett. Vol. 4, no. 6, pp. 1085-1088, 2004.

[3] S. I. Bozhevolnyi, V. S. Volkov, E. Devaux, J.-Y. Laluet, and T. W. Ebbesen, "Channel plasmon subwavelength waveguide components including interferometers and ring resonators," Nature, vol. 440, no. 7083, pp. 508-511, 2006.

[4] T.-B. Wang, X.-W. Wen, C.-P. Yin, and H.-Z. Wang, "The transmission characteristics of surface plasmon polaritons in ring resonator," Opt. Express, vol. 17, no. 26, pp. 24096-24101, 2009.

[5] C.-L. Zou, F.-W. Sun, C.-H. Dong, X.-F. Ren, J.-M. Cui, X.-D. Chen, Z.-F. Han, and G.-C. Guo, "Broadband integrated polarization beam splitter with surface plasmon," Opt. Lett. vol. 36, no. 18, pp. 3630-3632, 2011.

[6] G. Wang, H. Lu, X. Liu, D. Mao, and L. Duan, "Tunable multi-channel wavelength demultiplexer based on MIM plasmonic nanodisk resonators at telecommunication regime," Opt. Express, vol. 19, no. 4, pp. 3513-3518, 2011.

[7] S. Bashiri and K. Fasihi, "An all-optical $1 \times 2$ Demultiplexer Using Kerr Nonlinear Nano-plasmonic Switches,” Plasmonics, vol. 15, no. 2, pp. 449-456, 2020.

[8] F. J. Garcia de Abajo, "Graphene plasmonics: challenges and opportunities," ACS Photon. vol. 1, no. 3, pp. 135-152, 2014.

[9] F. Bonaccorso, Z. Sun, T. Hasan, and A. Ferrari, "Graphene photonics and optoelectronics," Nature Photon. vol. 4, no. 9, pp. 611, 2010.

[10] P. A. Huidobro, M. Kraft, R. Kun, S. A. Maier, and J. B. Pendry, "Graphene, plasmons and transformation optics," J. Opt. vol. 18, no. 4, pp. 044024, 2016.

[11] K. S. Novoselov and A. Geim, "The rise of graphene," Nat. Mater. vol. 6, no. 3, pp. 183-191, 2007.

[12] A. Vakil and N. Engheta, "Transformation optics using graphene," Science, vol. 332, no. 6035, pp. 1291-1294, 2011.

[13] C. Riedl, C. Coletti, and U. Starke, "Structural and electronic properties of epitaxial graphene on $\mathrm{SiC}\left(\begin{array}{llll}0 & 0 & 0 & 1\end{array}\right)$ : a review of growth, characterization, transfer doping and hydrogen intercalation," J. Phys. D: Appl. Phys. vol. 43, no. 37, pp. 374009, 2010. 
[14] F. Wang, Y. Zhang, C. Tian, C. Girit, A. Zettl, M. Crommie, and Y. R. Shen, "Gatevariable optical transitions in graphene," Science, vol. 320, no. 5873, pp. 206-209, 2008.

[15] M. Jablan, H. Buljan, and M. Soljačić, "Plasmonics in graphene at infrared frequencies," Phys. Rev. B, vol. 80, no. 24, pp. 245435, 2009.

[16] Y. Yao, M. A. Kats, P. Genevet, N. Yu, Y. Song, J. Kong, and F. Capasso, "Broad electrical tuning of graphene-loaded plasmonic antennas," Nano Lett. vol. 13, no. 3, pp. 1257-1264, 2013.

[17] Z. Wu, Y. Chen, T. Zhang, Z. Shao, Y. Wen, P. Xu, Y. Zhang, and S. Yu, "Design and optimization of optical modulators based on graphene-on-silicon nitride microring resonators," J. Opt. vol. 19, no. 4, pp. 045801, 2017.

[18] Z. Lu and W. Zhao, "Nanoscale electro-optic modulators based on graphene-slot waveguides," J. Opt. Soc. Am. B, vol. 29, no. 6, pp. 1490-1496, 2012.

[19] S. Bahadori-Haghighi, R. Ghayour, and M. H. Sheikhi, "Three-dimensional analysis of an ultrashort optical cross-bar switch based on a graphene plasmonic coupler," $J$. Lightwave Technol. vol. 35, no. 11, pp. 2211-2217, 2017.

[20] X. Liu, G Liu, P Tang, G Fu, G Du, Q Chen, and Z Liu, "Quantitatively optical and electrical-adjusting high-performance switch by graphene plasmonic perfect absorbers," Carbon, vol. 140, pp. 362-367, 2018.

[21] M. Janfaza, M. A. Mansouri-Birjandi, and A. Tavousi, "Proposal for a graphene nanoribbon assisted mid-infrared band-stop/band-pass filter based on bragg gratings," Opt. Commun. vol. 440, pp. 75-82, 2019.

[22] M. Janfaza, M. A. Mansouri-Birjandi, and A. Tavousi, "Tunable plasmonic band-pass filter based on Fabry-Perot graphene nanoribbons," Appl. Phys. B, vol. 123, no. 10, pp. $262,2017$.

[23] A. Tavousi, M. A. Mansouri-Birjandi, and M. Janfaza, "Optoelectronic application of graphene nanoribbon for mid-infrared bandpass filtering," App. Opt. vol. 57, no. 20, pp. 5800-5805, 2018.

[24] Y. Feng, Y. Liu, Y. Shi, X. Wang, and D. Dong, "An ultra-compact tunable intersection structure based on graphene nanoribbon," J. Phys. D: Appl. Phys. vol. 50, no. 18 , pp. $185101,2017$.

[25] J. Yang, H. Xin, Y. Han, D. Chen, J. Zhang, J. Huang, and Z. Zhang, "Ultra-compact beam splitter and filter based on a graphene plasmon waveguide," App. Opt. vol. 56, no. 35, pp. 9814-9821, 2017. 
[26] S. H. Lee, M Choi, T.-T. Kim, S. Lee, M. Liu, X. Yin. H. K. Choi, S. S. Lee, C.-G. Choi, and S.-Y. Choi, "Switching terahertz waves with gate-controlled active graphene metamaterials," Nature materials, vol. 11, no. 11, pp. 936-941, 2012.

[27] X. He, “Tunable terahertz graphene metamaterials," Carbon 82, 229-237, 2015.

[28] S. Asgari and M. Rahmanzadeh, "Tunable circular conversion dichroism and asymmetric transmission of terahertz graphene metasurface composed of split rings," Opt. Commun. vol. 456, pp. 124623, 2020.

[29] S.-F. Shi, B. Zeng, H.-L. Han, X. Hong, H.-Z. Tsai, H.-S. Jung, A. Zettl, M. F. Crommie, and F. Wang, "Optimizing broadband terahertz modulation with hybrid graphene/metasurface structures," Nano Lett. vol. 15, no. 1, pp. 372-377, 2015.

[30] X. Han, T. Wang, X. Li, S. Xiao, and Y. Zhu, "Dynamically tunable plasmon induced transparency in a graphene-based nanoribbon waveguide coupled with graphene rectangular resonators structure on sapphire substrate," Opt. Express, vol. 23, no. 25, pp. 31945-31955, 2015.

[31] M. Janfaza, M. A. Mansouri-Birjandi, and A. Tavousi, "Dynamic switching between single and double plasmon induced reflection through graphene nanoribbons based structure," Mater. Res. Express, vol. 5, no. 11, pp. 115022, 2018.

[32] M. Janfaza, M. A. Mansouri-Birjandi, and A. Tavousi, "Tunable plasmon-induced reflection based on graphene nanoribbon Fabry-Perot resonator and nanodisks," Opt. Mater. vol. 84, pp. 675-680, 2018.

[33] H. Zhuang, F. Kong, K. Li, and S. Sheng, "Plasmonic bandpass filter based on graphene nanoribbon," Appl. Opt. vol. 54, no. 10, pp. 2558-2564, 2015.

[34] S. He, X. Zhang, and Y. He, "Graphene nano-ribbon waveguides of record-small mode area and ultra-high effective refractive indices for future VLSI," Opt. Express, vol. 21, no. 25 , pp. 30664-30673, 2013.

[35] A. Nikitin, P. Alonso-González, S. Vélez, S. Mastel, A. Centeno, A. Pesquera, A. Zurutuza, F. Casanova, LE. Hueso, and FHL. Koppens, "Real-space mapping of tailored sheet and edge plasmons in graphene nanoresonators," Nature Photon. vol. 10, no. 4, pp. 239-243, 2016.

[36] Z. Fei, M. Goldflam, J.-S. Wu, S. Dai, M. Wagner, A. McLeod, MK. Liu, KW. Post, S. Zhu, and GCAM. Janssen, "Edge and surface plasmons in graphene nanoribbons," Nano Lett. vol. 15, no. 12, pp. 8271-8276, 2015.

[37] B.-Y. Jiang, E. J. Mele, and M. M. Fogler, "Theory of plasmon reflection by a 1D junction," Opt. Express, vol. 26, no. 13, pp. 17209-17226, 2018. 
[38] J.-H. Kang, S. Wang, and F. Wang, "Tunneling of two-dimensional surface polaritons through nanogaps in atomically thin crystals," Phys. Rev. B, vol. 99, no. 16, pp. $165408,2019$.

[39] E. D. Palik, Handbook of optical constants of solids (Academic, 1998), vol. 3.

[40] D. B. Farmer, D. Rodrigo, T. Low, and P. Avouris, "Plasmon-plasmon hybridization and bandwidth enhancement in nanostructured graphene," Nano lett. vol. 15, no. 4, pp. 2582-2587, 2015.

[41] D. Rodrigo, A. Tittl, O. Limaj, F. J. G. De Abajo, V. Pruneri, and H. Altug, "Doublelayer graphene for enhanced tunable infrared plasmonics," Light: Sci. Appl. vol. 6, no. 6, pp. e16277-e16277, 2017.

[42] H. Nasari and M. S. Abrishamian, "All-optical tunable notch filter by use of Kerr nonlinearity in the graphene microribbon array," J. Opt. Soc. Am. B, vol. 31, no. 7, pp. 1691-1697, 2014.

[43] L. Ju, G. Baisong, J. Horng, C. Girit, M. Martin, Z. Hao, H. A. Bechtel, X. Liang, A. Zettl, and Y. R. Shen, "Graphene plasmonics for tunable terahertz metamaterials," Nature nanotechno. vol. 6, no. 10, pp. 630-634, 2011.

[44] X. Li, W. Cai, J. An, S. Kim, J. Nah, D. Yang, R. Piner, A. Velamakanni, I. Jung, E. Tutuc, S. K. Banerjee, L. Colombo, and R. S. Ruoff, "Large area synthesis of high quality and uniform graphene films on copper foils," Science, vol. 324, no. 5932, pp. $1312-1314,2009$.

[45] P.-Y. Chen and A. Alu, "Atomically thin surface cloak using graphene monolayers," ACS Nano, vol. 5, no. 7, pp. 5855-5863, 2011.

[46] M. Maleki, M. Mehran, and A. Mokhtari, "Design of a near-infrared plasmonic gas sensor based on graphene nanogratings," J. Opt. Soc. Am. B, vol. 37, no. 11, pp. 3478$3486,2020$.

[47] B. Wang, X. Zhang, F. J. García-Vidal, X. Yuan, and J. Teng, "Strong coupling of surface plasmon polaritons in monolayer graphene sheet arrays," Phys. Rev. Lett. vol. 109, no. 7, pp. 073901, 2012.

[48] B. Wang, X. Zhang, X. Yuan, and J. Teng, "Optical coupling of surface plasmons between graphene sheets," Appl. Phys. Lett. vol. 100, no. 13, pp. 131111, 2012.

[49] H.-J. Li, L.-L. Wang, B. Sun, Z.-R. Huang, and X. Zhai, "Controlling mid-infrared surface plasmon polaritons in the parallel graphene pair," Appl. Phys. Express, vol. 7, no. 12, pp. 125101, 2014. 
[50] H.-J. Li, L.-L. Wang, Z.-R. Huang, B. Sun, and X. Zhai, "Tunable mid-infrared plasmonic anti-symmetric coupling resonator based on the parallel interlaced graphene pair," Plasmonics, vol. 10, no. 1, pp. 39-44, 2015.

[51] M.-D. He, K.-J. Wang, L. Wang, J.-B. Li, J.-Q. Liu, Z.-R. Huang, L. Wang, L. Wang, W.-D. Hu, and X. Chen, "Graphene-based terahertz tunable plasmonic directional coupler," Appl. Phys. Lett. vol. 105, no. 8, pp. 081903, 2014.

[52] Z.-R. Huang, L.-L. Wang, M.-D. He, H.-J. Li, B. Sun, J.-Q. Liu, and X. Zhai “An ultracompact tunable Bragg reflector based on edge propagating plasmons in graphene nanoribbon,” J. Modern Opt. vol. 62, no. 7, pp. 514-518, 2015.

[53] S. Zhang, W. Ji, R. Yin, X. Li, Z. Gong, and L. Lv, "Full bandwidth wavelength division multiplexer/demultiplexer based on MMI," IEEE Photon. Technol. Lett. vol. 30, no. 1, pp. 107-110, 2017. 


\section{Figures}

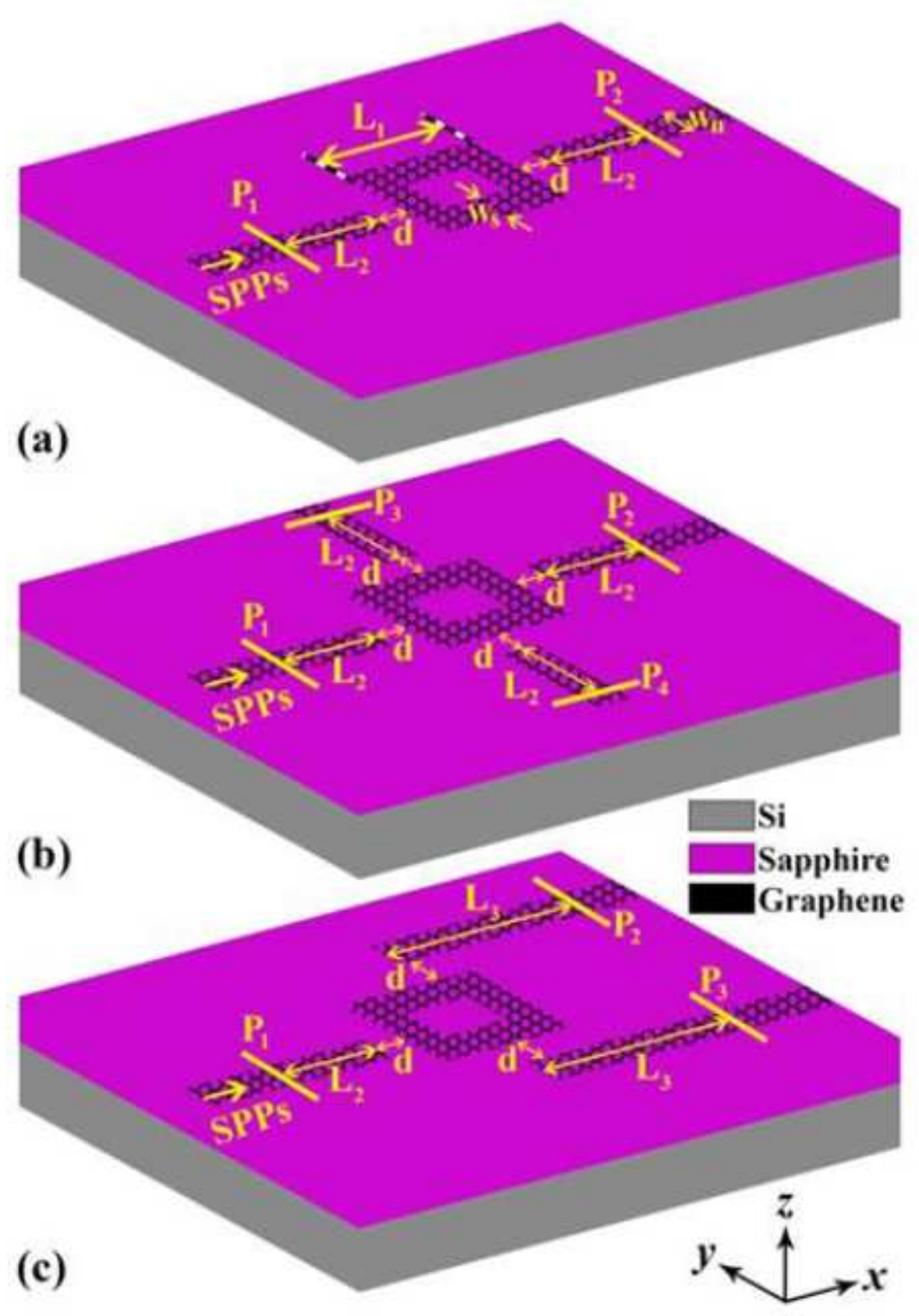

Figure 1

Three-Dimensional (3D) schematic diagram of the proposed structures based on GSNR for different applications (a) Band-pass filter. (b) Wavelength demultiplexer. (c) Power splitter. 

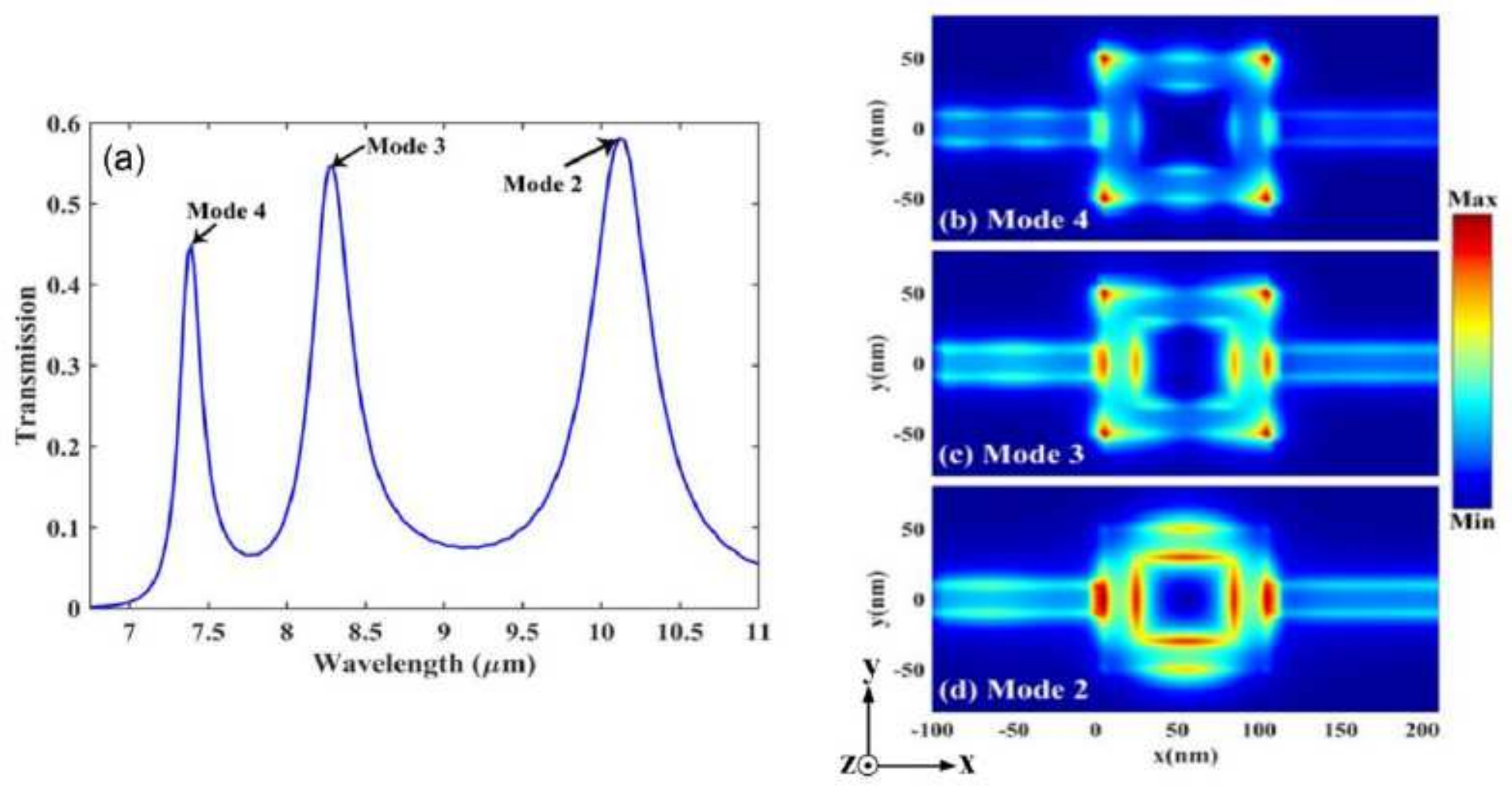

Figure 2

(a) The transmission spectrum of the proposed BPF structure is shown in Fig. 1(a). (b)-(d) Spatial distributions of $\mathrm{Hz}$ at the peaks of transmission for resonance modes. 

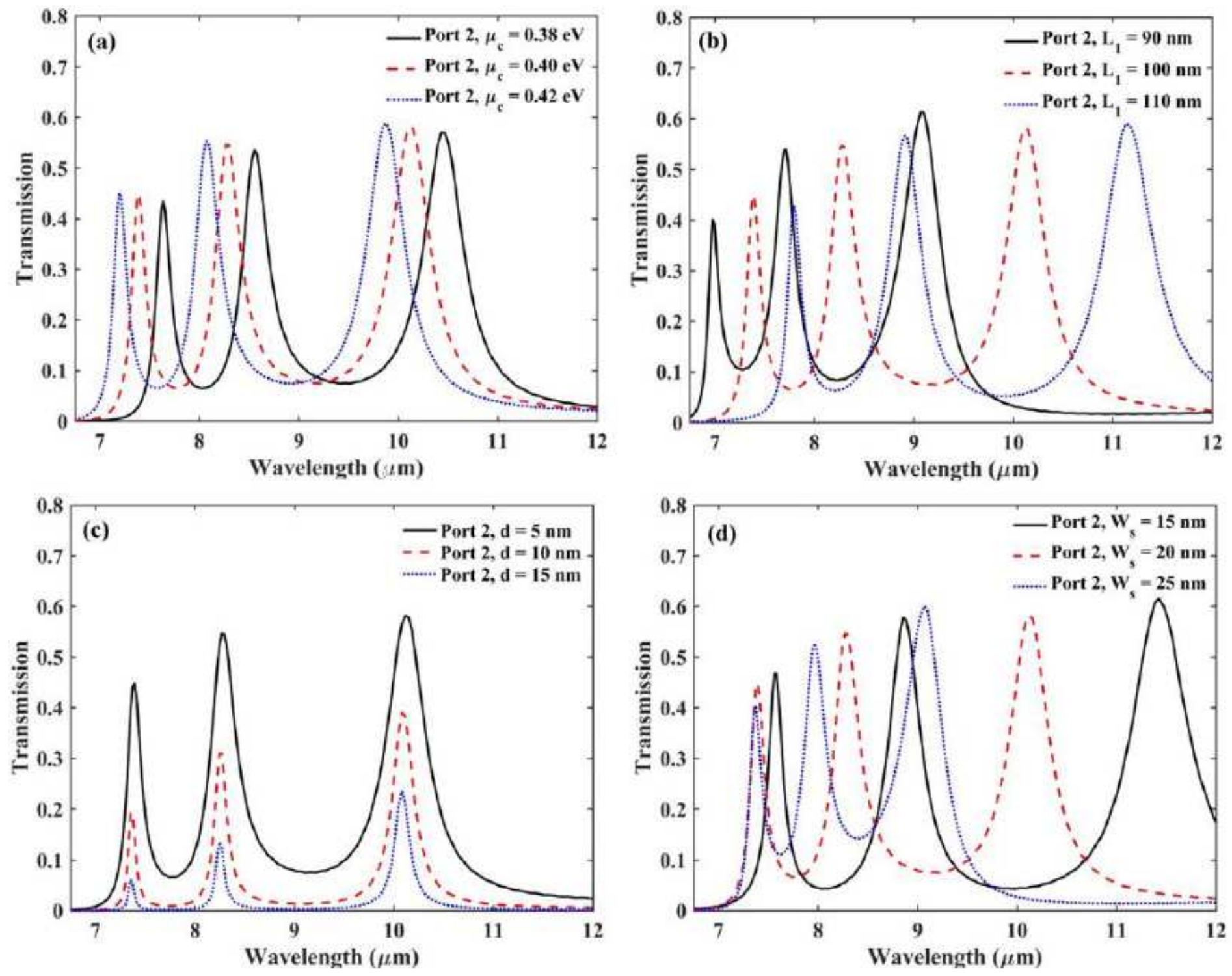

\section{Figure 3}

The transmission spectra of the proposed BPF structure are shown in Fig. 1(a) for different (a) $\mu \mathrm{c},(\mathrm{b})$ side lengths (L1) of GSNR, (c) coupling distance (d) between input/output GNR waveguides and GSNR, and (d) width (Ws) of GSNR. 

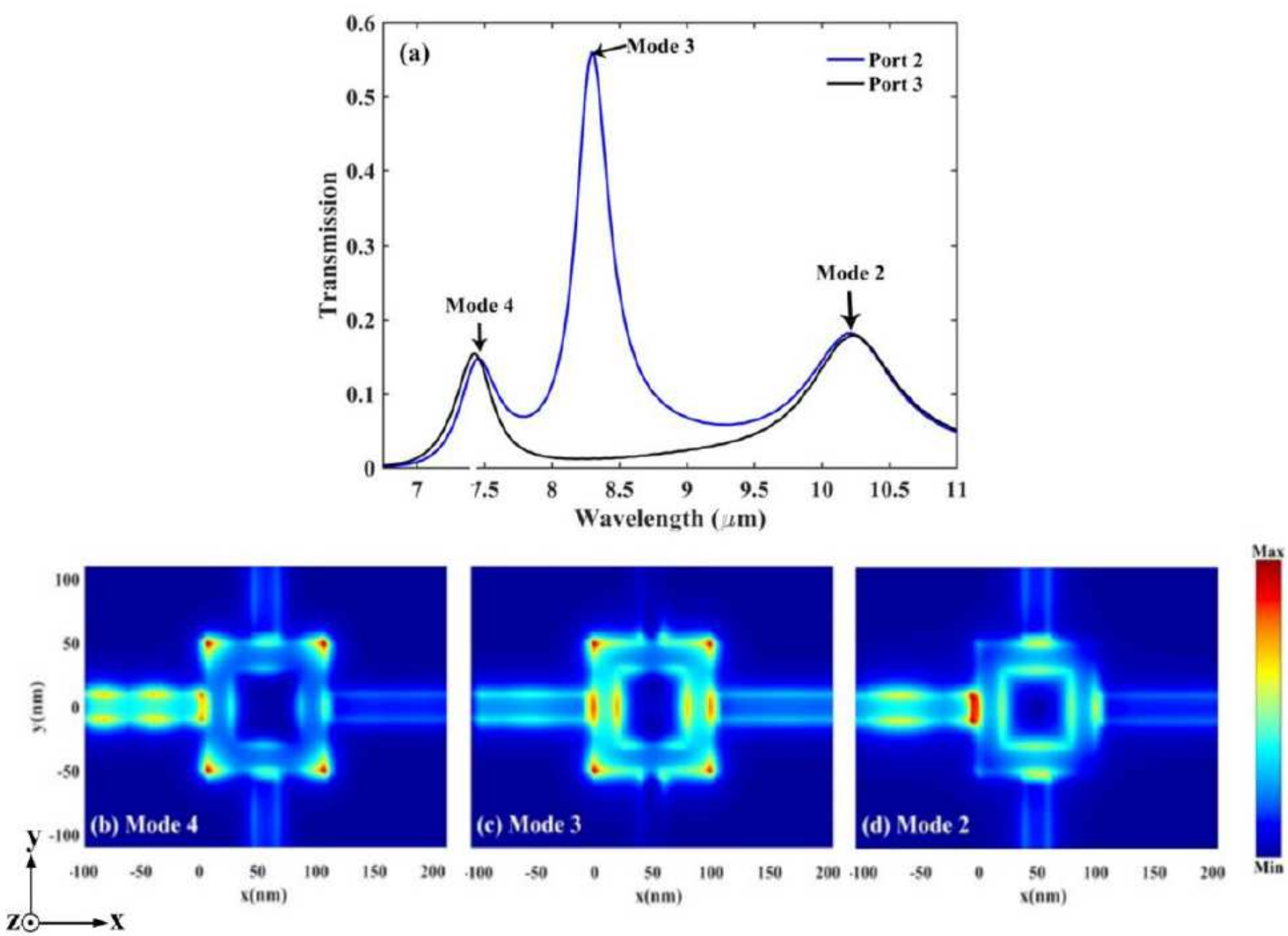

Figure 4

(a) The transmission spectra of ports 2 and 3 of the proposed wavelength demultiplexer structure are shown in Fig. 1(b). (b)-(d) The spatial distributions of $\mathrm{Hz}$ at the peaks of transmission for resonance modes. 

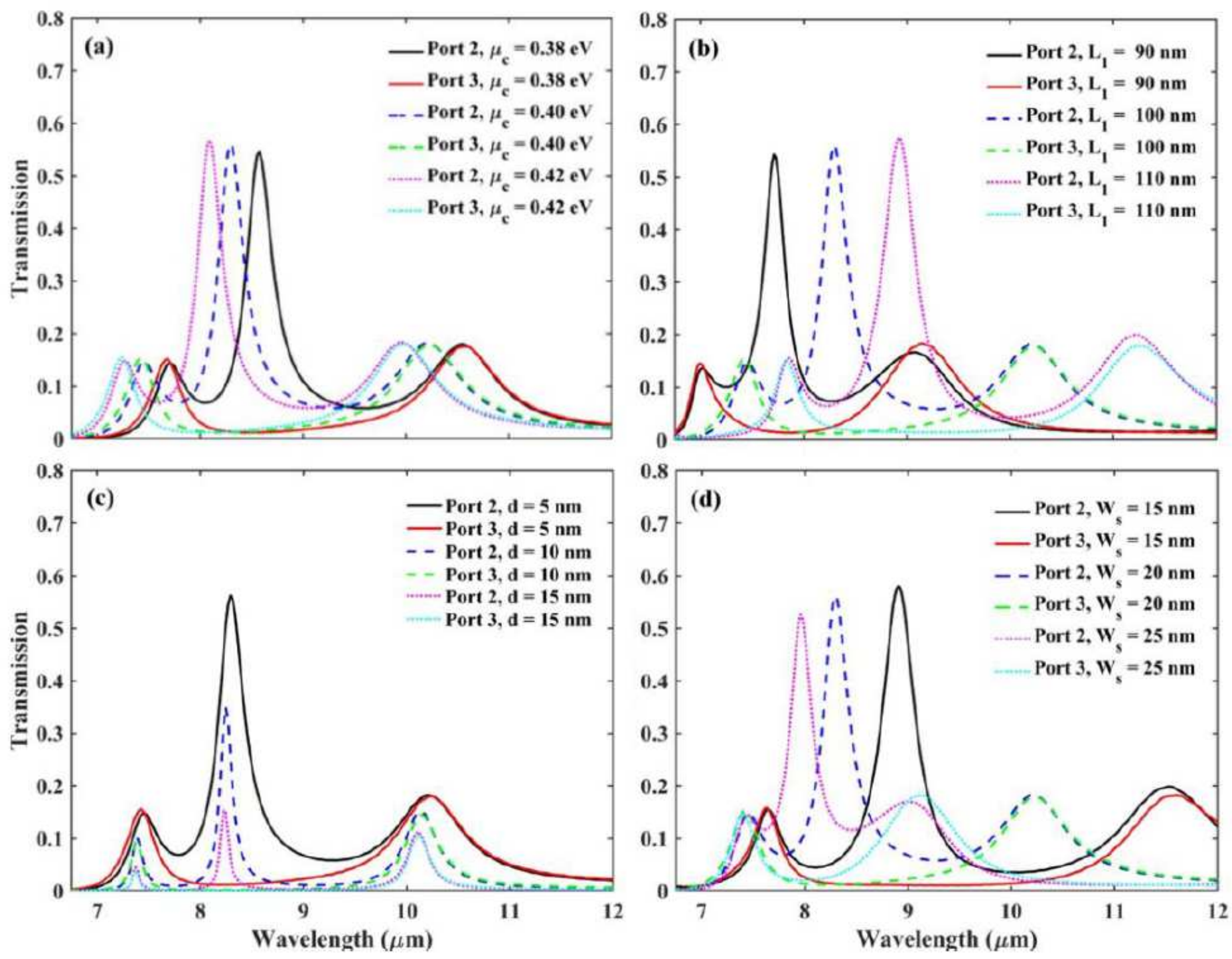

Figure 5

The transmission spectra of ports 2 and 3 of the proposed wavelength demultiplexer structure are shown in Fig. 1(b) for different (a) $\mu \mathrm{c}$, (b) side lengths (L1) of GSNR, (c) coupling distance (d) between input/output GNR waveguides and GSNR, and (d) width (Ws) of GSNR. 

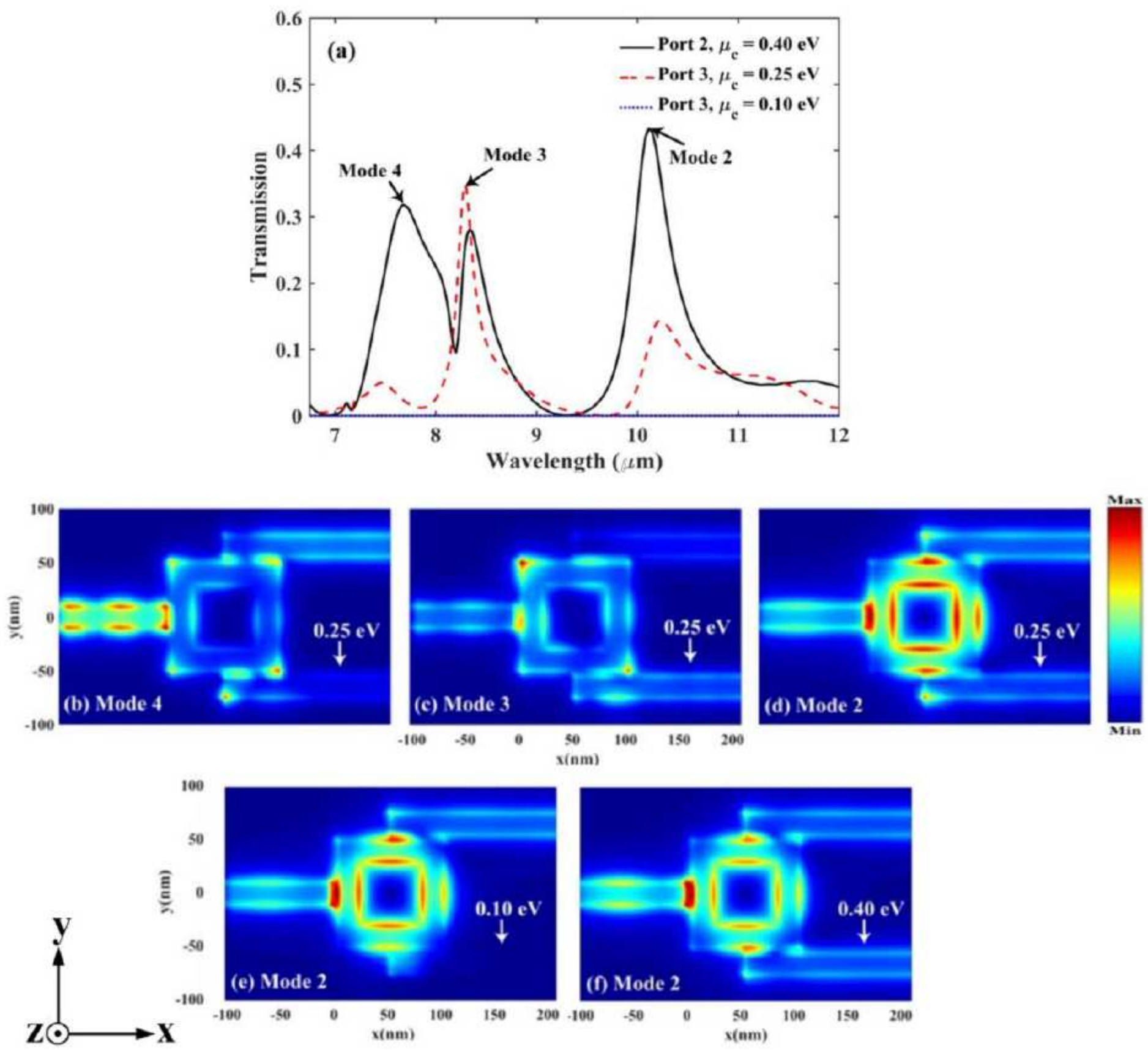

Figure 6

(a) The transmission spectra of ports 2 and 3 of the proposed power splitter structure are shown in Fig. 1(c) for different $\mu \mathrm{c}$ for port 3 and $\mu \mathrm{c}=0.40 \mathrm{eV}$ for port 2. (b)-(d) the spatial distributions of $\mathrm{Hz}$ for resonance modes with $\mu \mathrm{c}=0.25 \mathrm{eV}$ for port 3. (e) Spatial distribution of $\mathrm{Hz}$ for the mode 2 with $\mu \mathrm{c}=0.10$ $\mathrm{eV}$ for port 3. (f) Spatial distribution of $\mathrm{Hz}$ for the mode 2 with $\mu \mathrm{c}=0.40 \mathrm{eV}$ for both ports. 

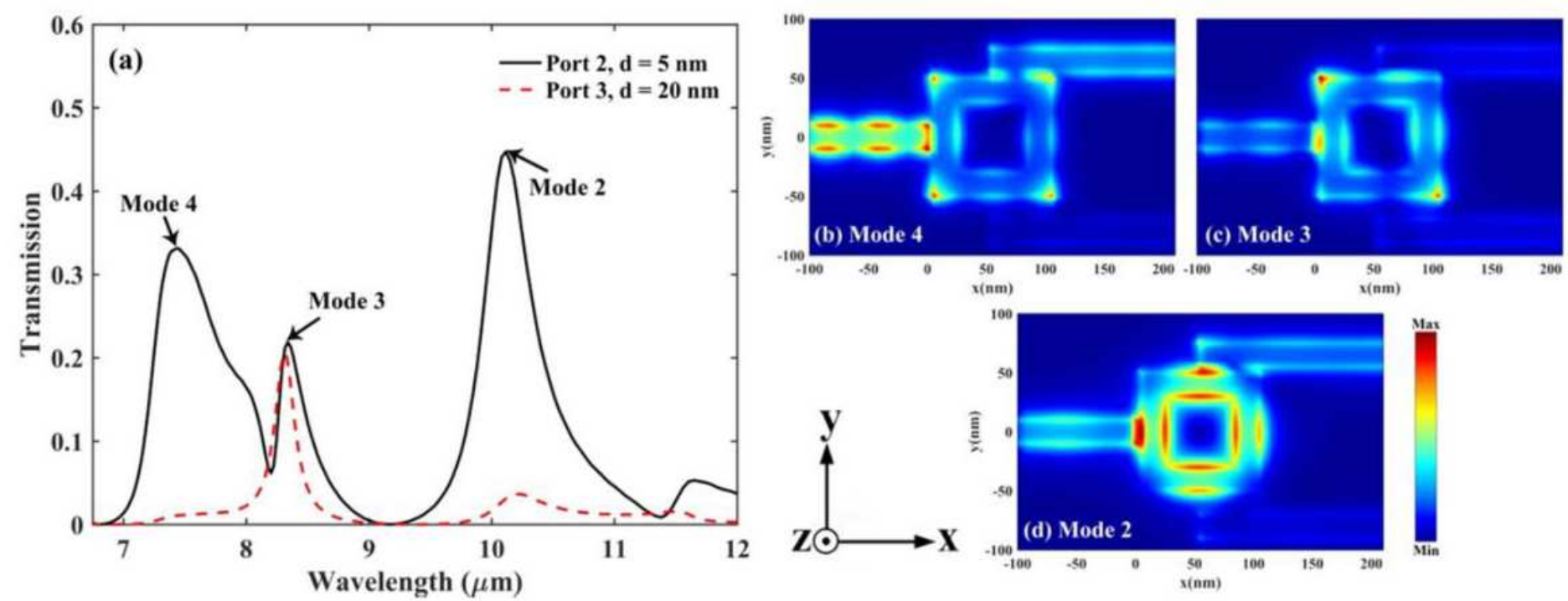

Figure 7

(a) The transmission spectra of ports 2 and 3 of the proposed power splitter structure are shown in Fig. 1(c) for different d. (b)-(d) The spatial distributions of $\mathrm{Hz}$ at the peaks of transmission for resonance modes. 